\title{
Objectifying Acupuncture Effects by Lung Function and Numeric Rating Scale in Patients Undergoing Heart Surgery
}

\author{
Anna Maimer, ${ }^{1,2,3}$ Andrew Remppis, ${ }^{4}$ Falk-Udo Sack, ${ }^{5}$ Stefanie Ringes-Lichtenberg, \\ Tobias Greten, ${ }^{2,3}$ Frank Brazkiewicz, ${ }^{2,3}$ Sven Schröder, ${ }^{2,3,6}$ Mario Goncalves, ${ }^{2,3}$ \\ Thomas Efferth, ${ }^{7}$ and Henry Johannes Greten ${ }^{2,3}$ \\ ${ }^{1}$ Department of Endocrinology and Metabolism, University of Heidelberg, Im Neuenheimer Feld 410, 69120 Heidelberg, Germany \\ ${ }^{2}$ Heidelberg School of Chinese Medicine, Karlsruher Straße 12, 69126 Heidelberg, Germany \\ ${ }^{3}$ ICBAS, University of Porto, Rua de Jorge Viterbo Ferreira No. 228, 4050-313 Porto, Portugal \\ ${ }^{4}$ Department of Cardiology, University of Heidelberg, Im Neuenheimer Feld 410, 69120 Heidelberg, Germany \\ ${ }^{5}$ Department of Heart Surgery, University of Heidelberg, Im Neuenheimer Feld 400, 69120 Heidelberg, Germany \\ ${ }^{6}$ HanseMerkur Center for Traditional Chinese Medicine, University Medical Center Hamburg-Eppendorf, Martinistraße 52, \\ 20246 Hamburg, Germany \\ ${ }^{7}$ Department of Pharmaceutical Biology, Institute of Pharmacy and Biochemistry, Johannes Gutenberg University of Mainz, \\ Staudinger Weg 5, 55099 Mainz, Germany
}

Correspondence should be addressed to Henry Johannes Greten; heidelbergschool@aol.com

Received 23 September 2012; Accepted 10 December 2012

Academic Editor: Lixing Lao

Copyright (C) 2013 Anna Maimer et al. This is an open access article distributed under the Creative Commons Attribution License, which permits unrestricted use, distribution, and reproduction in any medium, provided the original work is properly cited.

\begin{abstract}
Rationale. Poststernotomy pain and impaired breathing are common clinical problems in early postoperative care following heart surgery. Insufficiently treated pain increases the risk of pulmonary complications. High-dose opioids are used for pain management, but they may cause side effects such as respiratory depression. Study Design. We performed a prospective, randomized, controlled, observer-blinded, three-armed clinical trial with 100 patients. Group $1(n=33)$ and Group $2(n=34)$ received one 20 min session of standardized acupuncture treatment with two different sets of acupoints. Group $3(n=33)$ served as standard analgesia control without additional intervention. Results. Primary endpoint analysis revealed a statistically significant analgesic effect for both acupuncture treatments. Group 1 showed a mean percentile pain reduction (PPR) of 18\% (SD 19, $P<0.001)$. Group 2 yielded a mean PPR of $71 \%$ (SD 13, $P<0.001$ ). In Group 1, acupuncture resulted in a mean forced vital capacity (FVC) increase of $30 \mathrm{~cm}^{3}(\mathrm{SD}$ 73 ) without statistical significance $(P=0.303)$. In Group 2, posttreatment FVC showed a significant increase of $306 \mathrm{~cm}^{3}(\mathrm{SD} 215$, $P<0.001)$. Conclusion. Acupuncture revealed specific analgesic effects after sternotomy. Objective measurement of poststernotomy pain via lung function test was possible.
\end{abstract}

\section{Introduction}

Median sternotomy represents a surgical technique, in which the sternum is longitudinally incised and opened to get access to the organs of the mediastinum, for example, for heart transplantation, to set bypasses or valves on aortocoronary arteries, or to remove thymus tumors. Poststernotomy pain and impaired spontaneous breathing are common clinical problems in early postoperative care following heart surgery $[1,2]$. Insufficiently treated pain impedes coughing and physiological rib cage expansion and, thus, increases the risk of pulmonary complications such as atelectasis and pneumonia [2].

High-dose application of opioids represents the standard therapy after sternotomy. It is controversially discussed whether opioids after median sternotomy lead to side effects such as respiratory depression, vomiting, nausea, and sedation $[3,4]$. Therefore, combination therapy regimens have been applied containing opioids and traditional nonsteroidal anti-inflammatory drugs (tNSAIDs), cy- 
clooxygenase-2 (COX-2) inhibitors, alpha2-inhibitors, or other nonopioid drugs such as paracetamol. The effectiveness of these combinations in pain reduction is, however, critically discussed [5-7]. Therefore, there is an urgent need for the improvement of pain therapy after median sternotomy. It has been suggested that electroacupuncture might be a valuable adjunct in managing poststernotomy pain $[8,9]$. Whether needle acupuncture is a considerable asset in pain treatment after sternotomy is unknown as yet.

The aim of the present investigation was, therefore, to investigate the effect of needle acupuncture on pain-induced reduction of lung function after median sternotomy. To this end, a prospective, randomized, controlled, patient- and observer-blinded clinical trial was performed with patients undergoing median sternotomy.

\section{Methods}

The study was designed as a prospective, randomized, controlled, patient- and observer-blinded, three-armed clinical trail. Between September 2005 and August 2008, 100 thoracotomized patients were recruited from the intermediate intensive care unit (IMC) in the Department of Heart Surgery at Heidelberg University Hospital. Informed consent was obtained before subject enrollment according to a clinical trial protocol approved by the local Ethical Committee.

2.1. Inclusion and Exclusion Criteria. Patients were included in the study if they had undergone conventional on-bypass surgery via median sternotomy and reported pain during deep inspiration with an intensity of at least 3 on a $1-10$ numeric rating scale under standard analgesia with tNSARs and high-dose opioids. Patients had to be extubated and lucid. Patients meeting these criteria were excluded if they had a medical history of severe obstructive or restrictive pulmonary disease. Due to safety reasons, patients with a blood clotting time exceeding $4 \mathrm{~min}$ and/or a platelet count below $50.000 \mathrm{~nL}$ under anticoagulative therapy were also not accepted to the study. Additional ineligibility factors included acute general infection (fever, highly elevated leucocytes, or CRP) and/or local infection at the sites of potential needle insertion. Measures specifically influencing pain perception and/or lung function (i.e., inadvertent or accidental application of analgetics, physical exercise, physiotherapy treatment, and breathing exercises) were prohibited during the active $40 \mathrm{~min}$ study period $(10 \mathrm{~min}$ screening and randomization, $5 \mathrm{~min}$ baseline assessment, $20 \mathrm{~min}$ acupuncture/observation, and 5 min control assessment) and would result in an exclusion from the study. Furthermore, intolerance to acupuncture treatment or sudden worsening of the physical condition would lead to a discontinuation of the study.

2.2. Randomization to Study Groups. Subjects were randomized to 3 study groups: Group 1 and 2 received one single 20 min session of standardized verum acupuncture treatment in addition to standard analgesia. Acupuncture was performed on 12 acupoints using sterile $0.25 \times 40 \mathrm{~mm}$ surgical stainless steel needles. Needles were stimulated by rotation for approximately $5 \mathrm{sec}$ during insertion, after $10 \mathrm{~min}$, and before removal after $20 \mathrm{~min}$. Additionally, in both groups, six of the selected 12 acupoints were treated with "blood-letting" or "leopard spotting technique" using sterile $033 \mathrm{~mm} 29$-gauge needles. To rule out systematic acupuncturist-dependent fluctuations in overall treatment effects, all acupuncture treatments were carried out by the same physician, who had advanced training in traditional Chinese medicine (TCM).

The only systematic difference between acupuncture in Group 1 and Group 2 was the acupoint selection. In Group 2 , the acupoint selection was based upon common classical Chinese diagnostic features shown by an a priori investigated representative patient collective (10 patients with varied sex, age, and cultural background with postoperative chest pain following sternotomy). A short summary of the acupoint selection is given in the following paragraph. Specific diagnostic technical terms are quoted and described with an auxiliary Western medical explanation in brackets.

According to a well-known part of classical Chinese medical theory - the Shang Han Lun-which is systematized in the "Algor Laedens Theory" of the "Heidelberg model of TCM", severe postoperative pain arises from "xue stasis" (relative hypoxis of a tissue arising from a disrupted microcirculation) due to "post-traumatic algor" (reduced microcirculation of a tissue following a trauma due to vasospasmic reflexes and immunological reactions) [10]. SP 10 was included as a classical acupoint to counteract xue stasis [11]. The common "orb pattern" (specific neuroaffective pattern with defined physical signs) presented by the patient collective indicated "a syndrome of the closing principle"-the simultaneous occurrence of a "splendor yang state" (neuroimmunological reaction with signs of the "stomach" and the "large intestine" orb pattern) and a "yin flectens state" (neuroimmunological reaction with signs of the "pericardium" and "liver" orb pattern) [10]. Respectively, ST 34, ST 44, PC 6, and LIV 2 were chosen as potent acupoints on the aforementioned orb meridians [11]. As all patients showed signs of a distinct "yin deficiency" (a labile neurovegetative regulation due to an overall deficiency of functional tissue), K 3 was added [11].

Acupoint selection in Group 1 served as a verum acupuncture control as it presented a form of inferior acupuncture-acupoints were exclusively selected according to their ascribed therapeutical actions and medical indications propagated by the acupuncture literature. LI 4 was included as a well-known, analgesic acupoint [11]. GV 20 and Ex 1 are generally ascribed psychovegetatively relaxing effects, possibly enhancing overall analgesia [11]. BL 60 is indicated to relief sharp chest pain and to loosen tension in the back muscles, GB 8 is recommended to treat feeling of pressure in the epigastric and thoracic regions, SI 6 is commonly used to uncramp the entire musculoskeletal system, and ST 8 is used to treat dyspnea [11].

A detailed list of the selected acupoints is illustrated in Tables 1 and 2. Group 3 served as a standard treatment control without additional acupuncture intervention. After baseline assessment, patients assigned to Group 3 were advised to avoid physical or mental stress and rest tranquilly in bed for 20 min under the supervision of the study investigator. In 
TABLE 1: Acupoint selection in Group 1 (control acupuncture).

\begin{tabular}{|c|c|c|c|c|c|}
\hline Engl. abbr. & Latin abbr. & Latin notation & Chinese notation & Needling technique & Localisation \\
\hline LI 4 & IC 4 & Valles coniunctae & Hegu & blood-letting & bilateral \\
\hline SI 6 & IT 6 & Senectus felix & Yanglao & blood-letting & bilateral \\
\hline BL 60 & V 60 & Olympus & Kunlun & blood-letting & bilateral \\
\hline Ex 1 & Ex 1 & Atrium impressionis & Yintang & conventional needle insertion & median \\
\hline GV 20 & $\operatorname{Rg} 20$ & Conventus omnium & Baihui & conventional needle insertion & median \\
\hline GB 8 & F 8 & Apex auriculi & Erdian/Shuanijue & conventional needle insertion & bilateral \\
\hline ST 8 & S 8 & Retinens capitis & Touwei & conventional needle insertion & bilateral \\
\hline
\end{tabular}

TABLE 2: Acupoint selection in Group 2 (classical Chinese acupuncture).

\begin{tabular}{|c|c|c|c|c|c|}
\hline Engl. abbr. & Latin abbr. & Latin notation & Chinese notation & Needling technique & Localisation \\
\hline PC 6 & PC 6 & Clusa interna & Neiguan & blood-letting & bilateral \\
\hline ST 34 & S 34 & Monticuli septi & Liangqiu & blood-letting & bilateral \\
\hline SP 10 & $\mathrm{~L} 10$ & Mare xue & Xuehai & conventional needle insertion & bilateral \\
\hline ST 44 & S 44 & Vestibulum internum & Neiting & blood-letting & bilateral \\
\hline K 3 & R 3 & Rivulus major & Taixi & conventional needle insertion & bilateral \\
\hline LIV 2 & $\mathrm{H} 2$ & Interstitium ambulatorium & Xingjian & conventional needle insertion & bilateral \\
\hline
\end{tabular}

this way, Group 3 displayed the physiological fluctuations of pain perception and FVC over a 20 min interval without any medical intervention (observation).

Randomization was stratified using consecutively numbered envelopes, containing the details of allocation. The envelopes were filled and sealed prior to study enrolment by an independent assistant according to a password-protected, computer-generated randomisation list. The envelopes were opened in a row by the acupuncturist at the bedside after recruitment. The designated observer and those patients allocated to an intervention arm (Group 1 or 2) were blind to treatment. Patients were informed that the study aimed to compare two different acupuncture treatments with respect to their analgesic effect after sternotomy.

2.3. Efficacy Parameters. Baseline and control efficacy assessment was carried out by a blinded observer and comprised two elements.

(1) Dynamic pain assessment via numeric rating scale to record changes in subjective pain perception was carried out dynamically-during deep inspiration. Two different numeric rating scales were chosen; to semiquantify baseline chest pain, a conventional 110 NRS was used. To record the residual chest pain after treatment/observation in relation to the initial pain intensity, a $0 \%-100 \%$ NRS (subdivided into $10 \%$ sections) was implemented. The baseline pain intensity served as the individual " $100 \%$-reference pain" for this $0 \%-100 \%$ NRS.

(2) Measurement of forced vital capacity (FVC) served as a physical and, thus, objective parameter to indicate analgesia-related functional improvements in rib cage expansion.

Percentile pain reduction (PPR) after treatment served as primary efficacy parameter, posttreatment change in FVC compared to baseline FVC was chosen as secondary efficacy parameter. For primary and secondary endpoint analyses, the Kruskal-Wallis test was performed as a 3-group global test, followed by the Wilcoxon test for post hoc analysis in case of a significant outcome. Correlation and subgroup analysis was performed to further investigate the a priori assumed pathophysiological connection between chest pain intensity and FVC measurement.

\section{Results}

Out of 367 screened patients, 100 patients met the inclusion criteria and were randomized to one out of three possible study groups. Participant flow through the trial is shown in Figure 1. Randomization resulted in an equal distribution of sociodemographic and clinical characteristics (see Table 3).

Primary endpoint analysis revealed a statistically significant analgesic effect for both acupuncture treatmentsacupuncture in Group 1 resulted in a mean PPR of $18 \%$ (SD 19, $P<0.001$ ), and acupuncture treatment in Group 2 yielded a mean PPR of $71 \%$ (SD 13, $P<0.001$ ). Figure 2 displays the distribution of PPR outcomes.

In Group 1, acupuncture resulted in a mean FVC increase of $30 \mathrm{~cm}^{3}$ (SD 73), which failed to attain statistical significance (1 versus 3: $P$-value $=0.303$ ). In Group 2, post-treatment FVC showed a statistically significant, average increase of $306 \mathrm{~cm}^{3}$ (SD 215, 2 versus 3: $P$-value $<0.001$ ), with a strong correlation to PPR outcomes (spearman's rank correlation coefficient $=0.74)$. Pain reduction and FVC changes after acupuncture in Group 1 did not correlate in a statistically significant manner $(P=0.503)$. Figure 3 illustrates the distribution of FVC-changes, and Figure 4 displays the correlation of PPR and FVC-changes in Group 2.

Subgroup analysis revealed that meaningful FVC increases $\left(\geq 300 \mathrm{~cm}^{3}\right)$ exclusively occurred in patients with a PPR of $60 \%$, or greater (see Figure 5). Below a PPR of $60 \%$, 
TABle 3: Patients' characteristics at baseline.

\begin{tabular}{|c|c|c|c|c|c|}
\hline & Group 1 & Group 2 & Group 3 (control) & Total & $P$ value \\
\hline$N$ & 33 & 34 & 33 & 100 & \\
\hline Male gender* & $24(73 \%)$ & $22(65 \%)$ & $26(79 \%)$ & $72(72 \%)$ & $0.436^{\infty}$ \\
\hline \multicolumn{5}{|l|}{ Age (years) } & \multirow[t]{2}{*}{$0.295^{\#}$} \\
\hline Mean (SD) & $65(10)$ & $68(11)$ & $66(10)$ & $66(10)$ & \\
\hline \multicolumn{5}{|l|}{ BMI $\left(\mathrm{kg} / \mathrm{m}^{2}\right)$} & \multirow{2}{*}{$0.515^{\#}$} \\
\hline Mean (SD) & $28(4)$ & $29(5)$ & $29(4)$ & $28(4)$ & \\
\hline \multicolumn{6}{|l|}{ Operation type } \\
\hline On-bypass* & $33(100 \%)$ & $34(100 \%)$ & $33(100 \%)$ & $100(100 \%)$ & \\
\hline Coronary revascularisation ${ }^{*}$ & $17(51 \%)$ & $20(58 \%)$ & $21(63 \%)$ & $58(58 \%)$ & \\
\hline Valve reconstruction or replacement ${ }^{*}$ & $15(45 \%)$ & $14(41 \%)$ & $15(45 \%)$ & $44(44 \%)$ & \\
\hline Aortic reconstruction after dissection ${ }^{*}$ & $1(3 \%)$ & $0(0 \%)$ & $0(\%)$ & $1(1 \%)$ & \\
\hline \multicolumn{6}{|l|}{ Chest pain baseline (0-10 NRS) } \\
\hline Median (IQR) & $6(2)$ & $5(2)$ & $5(2)$ & $5(2)$ & \multirow{2}{*}{$0.653^{\#}$} \\
\hline Mean (SD) & $5.5(1.2)$ & $5.2(1.4)$ & $5.3(1.3)$ & $5.3(1.3)$ & \\
\hline \multicolumn{6}{|l|}{ FVC baseline $\left(\mathrm{cm}^{3}\right)$} \\
\hline Median (IQR) & $1200(600)$ & $1000(550)$ & $1200(400)$ & $1150(600)$ & \multirow{2}{*}{$0.082^{\#}$} \\
\hline Mean (SD) & $1294(426)$ & $1147(405)$ & $1275(325)$ & $1238(390)$ & \\
\hline
\end{tabular}

* The numbers in parentheses display the percentage for dichotomous variables.

${ }^{\#} P$ values were calculated using the Kruskal-Wallis test.

${ }^{\infty} P$ values were calculated using the $\chi^{2}$-test.

Abbreviations-SD: standard deviation; IQR: interquartile range.

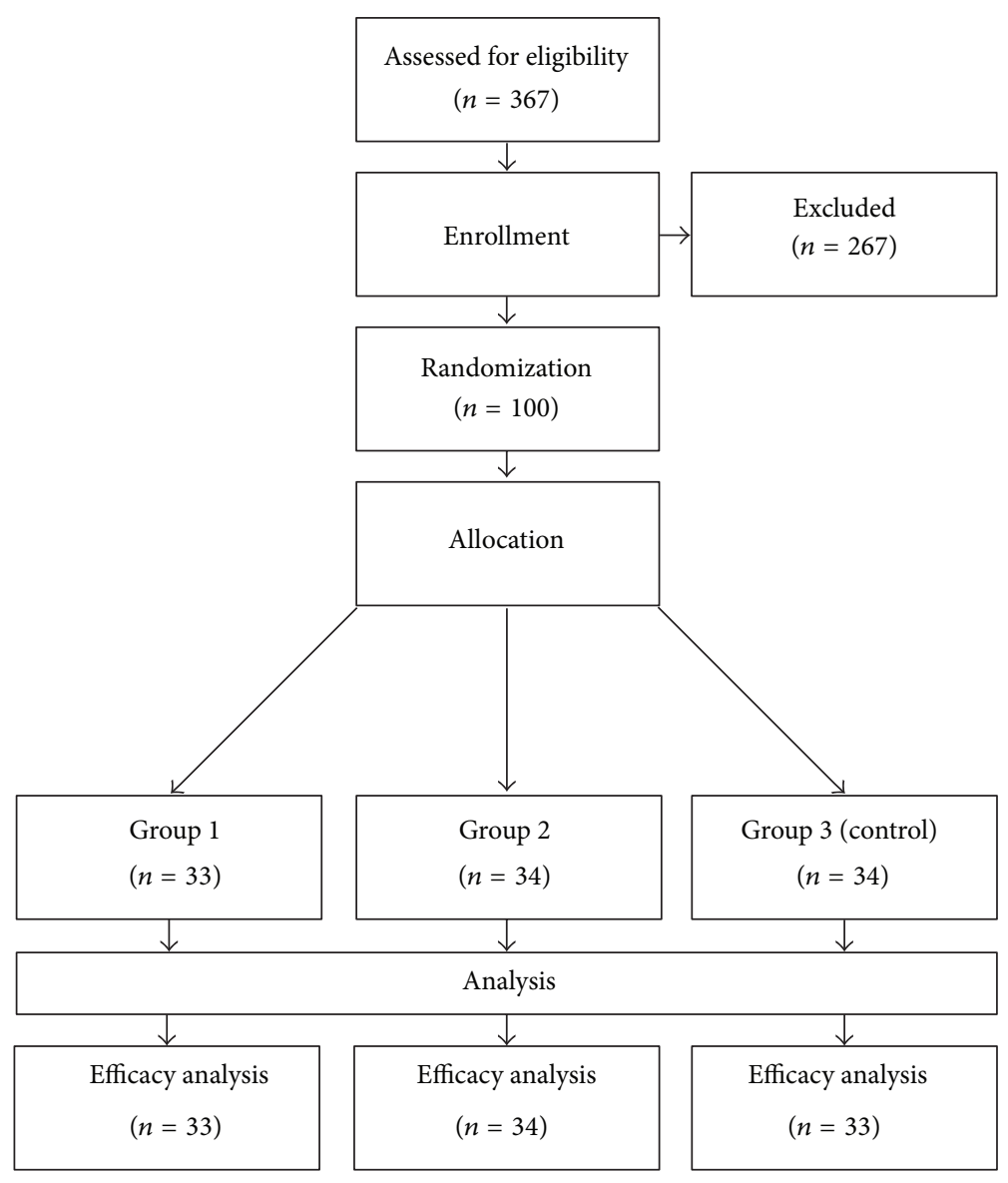

Figure 1: Participant flow. 


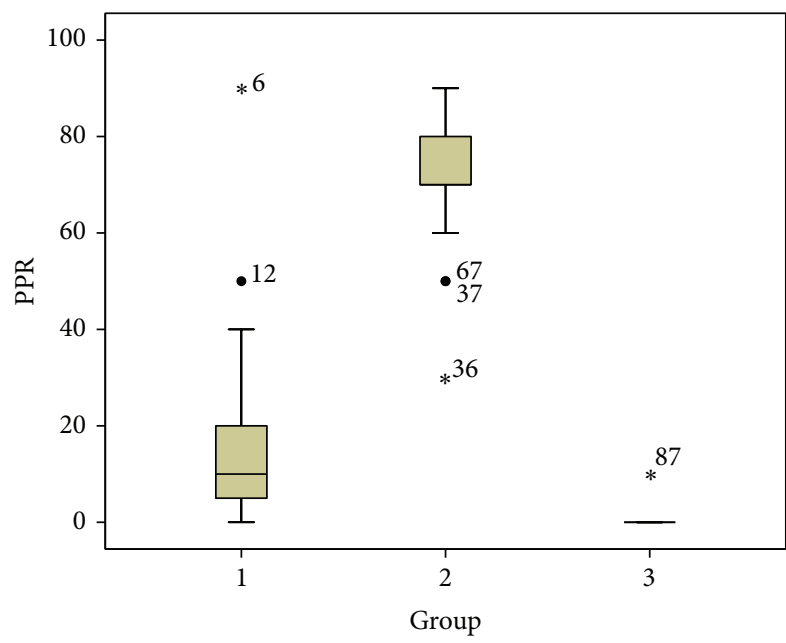

FIgURE 2: Distribution of PPR. Box-and-whisker plots represent lower quartile, median, upper quartile, maximum, minimum, outliers, and extreme values.

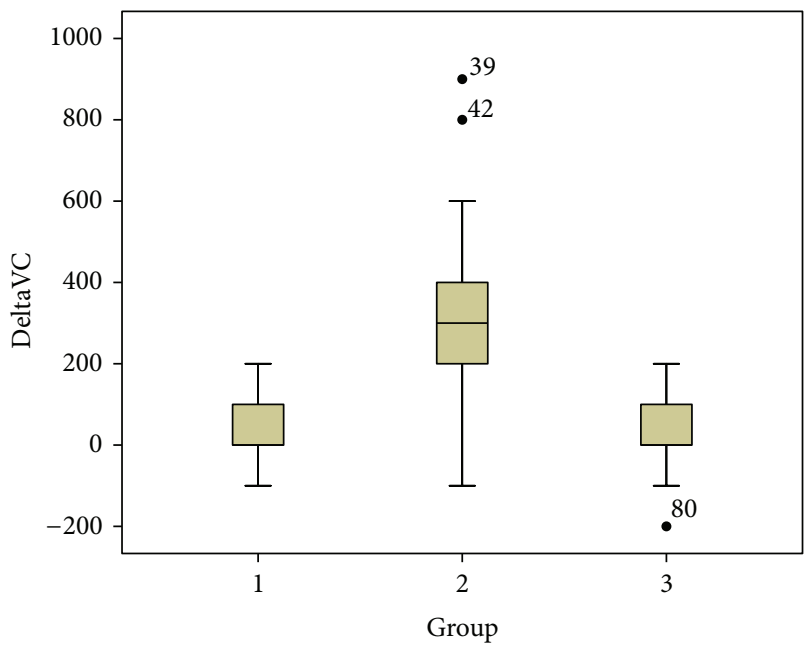

FIGURE 3: Distribution of FVC changes from baseline FVC. Boxand-whisker plots represent lower quartile, median, upper quartile, maximum, minimum, outliers, and extreme values.

the observed changes in FVC remained within the range of normal, acupuncture-independent fluctuations as shown by Group 3 patients.

\section{Discussion}

In the present investigation, we demonstrated statistically significant differences in immediate analgesic and functional effects between two types of acupuncture treatments compared to a control group in poststernotomy patients. The clinical trial showed that a lung function test can be used as an objective measurement of poststernotomy pain. Interestingly, only clinically significant analgesic effects $(\mathrm{PPR} \geq 60 \%)$ led to functional improvements in lung function. This result further emphasizes the importance of maximal efficacy of postoperative analgesia to prevent pulmonary complications

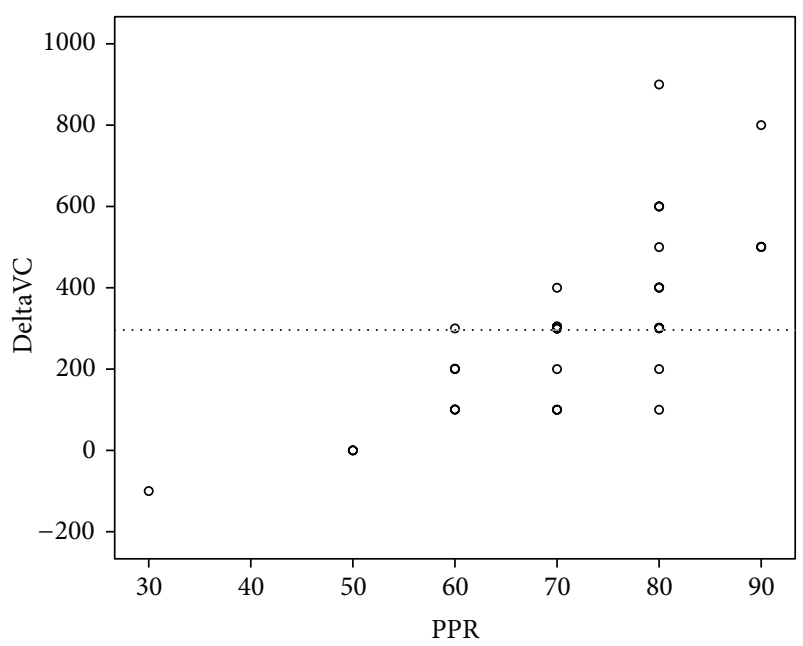

FIGURE 4: Correlation between PPR and posttreatment FVC changes in Group 2. Circles that are more intensely marked represent coordinates that occurred several times. The dotted line represents the lower limit for meaningful FVC changes $\left(\geq 300 \mathrm{~cm}^{3}\right)$.

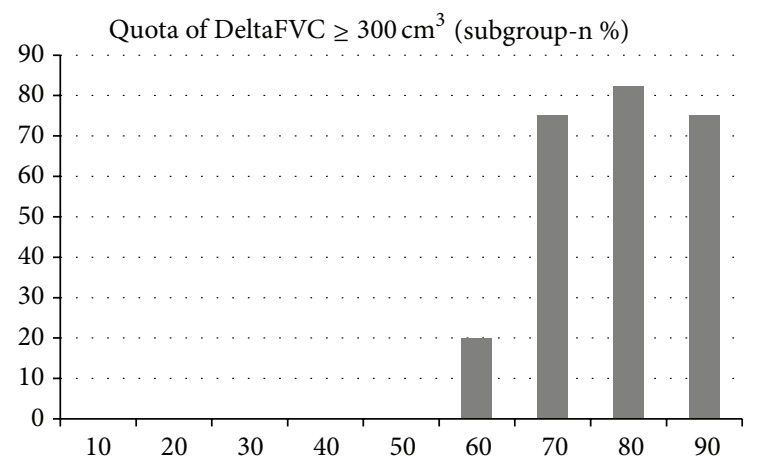

FIgURE 5: Subgroup analysis. Quota of clinically significant FVC increases $\left(+\geq 300 \mathrm{~cm}^{3}\right)$ in the individual PPR subgroups of Group 2.

[2]. Some limitations of the present study may be discussed. The study period was short with no repeated acupuncture treatments, and the results only mirror immediate analgesic effects. Further studies may be performed in the future to address these issues. A Blinding of acupuncturists did not take place, because this is merely achievable. Furthermore, no placebo control was implemented in the study, since from an ethical point of view, a nontreatment group is not justifiable in patients suffering from severe pain.

It is a long-lasting discussion in the scientific literature that strong postoperative pain and a considerable incidence of chronic pain after cardiac surgery and median sternotomy necessitate effective pain management [7]. A number of opioid-based treatment strategies have been described, including thoracic epidural anesthesia, spinal and intrathecal anesthesia, intercostal and paraventral blocks, or patientcontrolled intravenous analgesia (PCS) [7]. In addition to 
combining opioids with tNSAIDs, COX-2 inhibitors, alpha2inhibitors, or other drugs, several nonpharmacological strategies have been reported. Preoperative pain education of patients about postoperative pain resulted in less concerns about pain management [12]. Interestingly, electrical skin stimulation reduces pain perception of the organism. This technique was termed transcutaneous electrical nerve stimulation (TENS), which was successfully applied for poststernotomy pain management in several randomized clinical trials [13-15]. The stimulation of skin for pain reduction indicates that acupuncture as a technique applied since ages to treat pain associated with many diseases and symptoms may also be helpful for poststernotomy pain management. Indeed, electroacupuncture has been reported to reduce poststernotomy pain and to improve pulmonary function $[8,9]$. Electroacupuncture is based on the insertion of needles at specific acupoints together with electric current.

In the present investigation, we clearly demonstrated that classical needle acupuncture without electrostimulation also led to significant pain reduction and lung function improvement. This may have impact on future concepts of pain management after median sternotomy in heart surgery. The fact that the acupuncture treatment of Groups 1 and 2 differ in their analgesic effects in the present study indicates that it considerably matters which acupoints are used and contradicts the view that acupuncture might only mediate nonspecific skin stimulation, which may or may not exceed placebo effects [16]. As imaging studies have delivered evidence of acupoint-specific functional magnetic resonance imaging patterns $[17,18]$ and the selection of acupoints presented the only systematic difference between acupuncture treatment in Groups 1 and 2, the observable difference in analgesic effects can be attributed to the summation effect of all twelve individual acupoint-specific reactions. Furthermore, it can be argued that the diagnosis-dependent selection of acupoints has significantly contributed to the analgesic superiority observed in Group 2. If acupoints are understood as reflex points that elicit specific neurovegetative alterations, it can be speculated that they might only yield satisfactory treatment results if they match the current vegetative status of a patient (which is expressed in the TCM diagnosis) [10]. However, it is also imaginable that psychological confounders may have distorted post-treatment pain assessment; minimal to moderate pain reductions - as frequently observed in Group 1-are possibly underrated if patients were disappointed by the acupuncture treatment effect, while patients, who were positively surprised by the analgesic effect of acupuncture treatment, might tend to overrate its effect.

The exact mechanisms for acupuncture-mediated pain reduction in general and after median sternotomy are not well understood as yet. Different modes of action may account for pain reduction such as the release of endogenous opioids [7], adenosine A1 receptor-mediated antinociceptive effects [19], mast cell degranulation, and and the release of substance $P$, amongst others [20-26].

In conclusion, the present clinical trial demonstrated that acupuncture revealed specific effects in pain management after median sternotomy. Objective measurement of poststernotomy pain by a lung function test was possible and enabled to distinguish between functionally insignificant pain reduction (resulting in no improvement of breathing) and clinically significant analgesia effects of acupuncture.

\section{References}

[1] P. Lahtinen, H. Kokki, and M. Hynynen, "Pain after cardiac surgery: a prospective cohort study of 1-year incidence and intensity," Anesthesiology, vol. 105, no. 4, pp. 794-800, 2006.

[2] C. Weissman, "Pulmonary complications after cardiac surgery," Seminars in Cardiothoracic and Vascular Anesthesia, vol. 8, no. 3, pp. 185-211, 2004.

[3] T. Bouillon, J. Bruhn, H. Roepcke, and A. Hoeft, "Opioidinduced respiratory depression is associated with increased tidal volume variability," European Journal of Anaesthesiology, vol. 20, no. 2, pp. 127-133, 2003.

[4] D. Bainbridge, J. E. Martin, and D. C. Cheng, "Patientcontrolled versus nurse-controlled analgesia after cardiac surgery: a meta-analysis," Canadian Journal of Anesthesia, vol. 53, no. 5, pp. 492-499, 2006.

[5] E. Ott, N. A. Nussmeier, P. C. Duke et al., "Efficacy and safety of the cyclooxygenase 2 inhibitors parecoxib and valdecoxib in patients undergoing coronary artery bypass surgery," Journal of Thoracic and Cardiovascular Surgery, vol. 125, no. 6, pp. 14811492, 2003.

[6] C. Remy, E. Marret, and F. Bonnet, "Effects of acetaminophen on morphine side-effects and consumption after major surgery: meta-analysis of randomized controlled trials," British Journal of Anaesthesia, vol. 94, no. 4, pp. 505-513, 2005.

[7] M. Mazzeffi and Y. Khelemsky, "Poststernotomy pain: a clinical review," Journal of Cardiothoracic and Vascular Anesthesia, vol. 25, no. 6, pp. 1163-1178, 2011.

[8] W. Pongratz, W. Linke, and J. A. Richter, "Electroacupuncture analgesia in 500 heart surgery cases," Anasthesiol Intensivmed Praxis, vol. 13, no. 1, pp. 19-33, 1977 (German).

[9] M. C. Colak, A. Kavakli, A. Kilinç, and A. Rahman, "Postoperative pain and respiratory function in patients treated with electroacupuncture following coronary surgery," Neurosciences, vol. 15, no. 1, pp. 7-10, 2010.

[10] H. J. Greten, Extima/Intima: The Model of Stages of Cyclic Diseases. Understanding and Applying TCM, Georg Thieme, Stuttgart, Germany, 2nd edition, 2006.

[11] C.-H. Hempen and V. Wortmann Chow, Pocket Atlas of Acupuncture, Georg Thieme, Stuttgart, Germany, 2006.

[12] J. Watt-Watson, B. Stevens, J. Costello, J. Katz, and G. Reid, "Impact of preoperative education on pain management outcomes after coronary artery bypass graft surgery: a pilot," Canadian Journal of Nursing Research, vol. 31, no. 4, pp. 41-56, 2000.

[13] M. Emmiler, O. Solak, C. Kocogullari et al., "Control of acute postoperative pain by transcutaneous electrical nerve stimulation after open cardiac operations: a randomized placebocontrolled prospective study," The Heart Surgery Forum, vol. 11, no. 5, pp. E300-E303, 2008.

[14] G. Cipriano Jr., A. C. de Camargo Carvalho, G. F. Bernardelli, and P. A. T. Peres, "Short-term transcutaneous electrical nerve stimulation after cardiac surgery: effect on pain, pulmonary function and electrical muscle activity," Interactive Cardiovascular and Thoracic Surgery, vol. 7, no. 4, pp. 539-543, 2008.

[15] O. Solak, M. Emmiler, Y. Ela et al., "Comparison of continuous and intermittent transcutaneous electrical nerve stimulation in 
postoperative pain management after coronary artery bypass graft ing: a randomized, placebo-controlled prospective study," Heart Surgery Forum, vol. 12, no. 5, pp. E266-E271, 2009.

[16] A. Schneider, K. Streitberger, and S. Joos, "Acupuncture treatment in gastrointestinal diseases: a systematic review," World Journal of Gastroenterology, vol. 13, no. 25, pp. 3417-3424, 2007.

[17] B. Yan, K. Li, J. Xu et al., "Acupoint-specific fMRI patterns in human brain," Neuroscience Letters, vol. 383, no. 3, pp. 236-240, 2005.

[18] A. Campbell, "Point specificity of acupuncture in the light of recent clinical and imaging studies," Acupuncture in Medicine, vol. 24, no. 3, pp. 118-122, 2006.

[19] N. Goldman, M. Chen, T. Fujita et al., "Adenosine A1 receptors mediate local anti-nociceptive effects of acupuncture," Nature Neuroscience, vol. 13, no. 7, pp. 883-888, 2010.

[20] Y. M. Yang and P. P. Waug, "Morphological observation on the effect of acupuncture on mast cells at the zusanli point," Zhen ci yan jiu, vol. 11, no. 4, pp. 298-302, 1986 (Chinese).

[21] Z. Zhu and R. Xu, "Morphometric observation on the mast cells under the acupuncture meridian lines," Zhen ci yan jiu, vol. 15, no. 2, pp. 157-158, 1990 (Chinese).

[22] M. Kakurai, T. Demitsu, N. Umemoto, M. Ohtsuki, and H. Nakagawa, "Activation of mast cells by silver particles in a patient with localized argyria due to implantation of acupuncture needles," British Journal of Dermatology, vol. 148, no. 4, p. 822, 2003.

[23] D. Zhang, G. Ding, X. Shen et al., "Role of mast cells in acupuncture effect: a pilot study," Explore, vol. 4, no. 3, pp. 170177, 2008.

[24] M. Xiao-Peng, L. Y. Tan, Y. Yang et al., "Effect of electroacupuncture on substance $P$, its receptor and corticotropinreleasing hormone in rats with irritable bowel syndrome," World Journal of Gastroenterology, vol. 15, no. 41, pp. 5211-5217, 2009.

[25] K. Cheng, X. Y. Shen, G. H. Ding, and F. Wu, "Relationship between laser acupuncture analgesia and the function of mast cells," Zhongguo zhen jiu, vol. 29, no. 6, pp. 478-483, 2009 (Chinese).

[26] H. Shi, B. Cheng, J. H. Li et al., "Mast cell and substance P are involved in the process of acupoint sensitization induced by acute gastric mucosal injury," Zhen ci yan jiu, vol. 35, no. 5, pp. 323-329, 2010 (Chinese). 


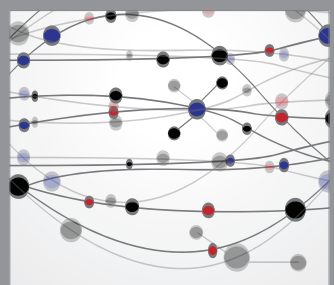

The Scientific World Journal
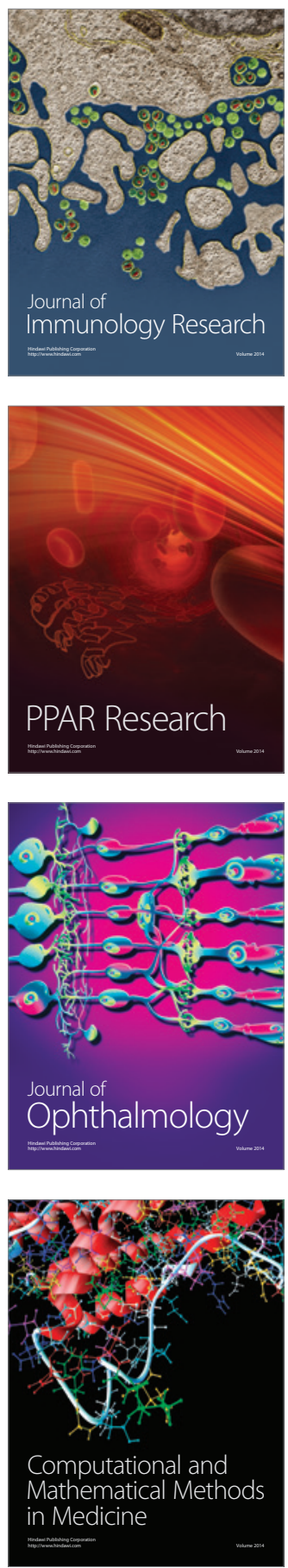

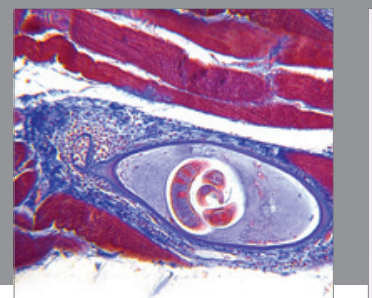

Gastroenterology

Research and Practice
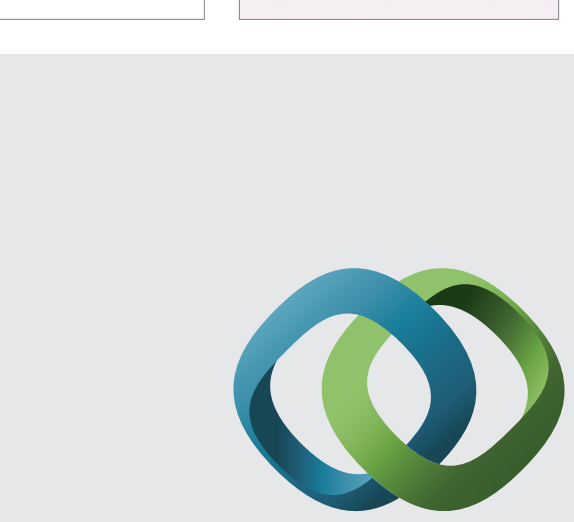

\section{Hindawi}

Submit your manuscripts at

http://www.hindawi.com
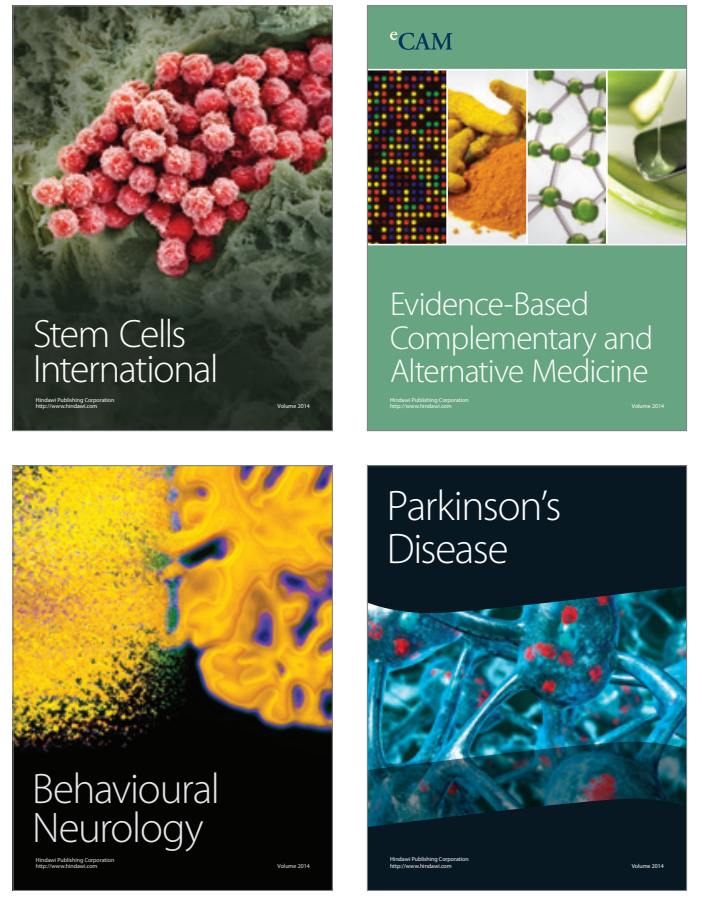
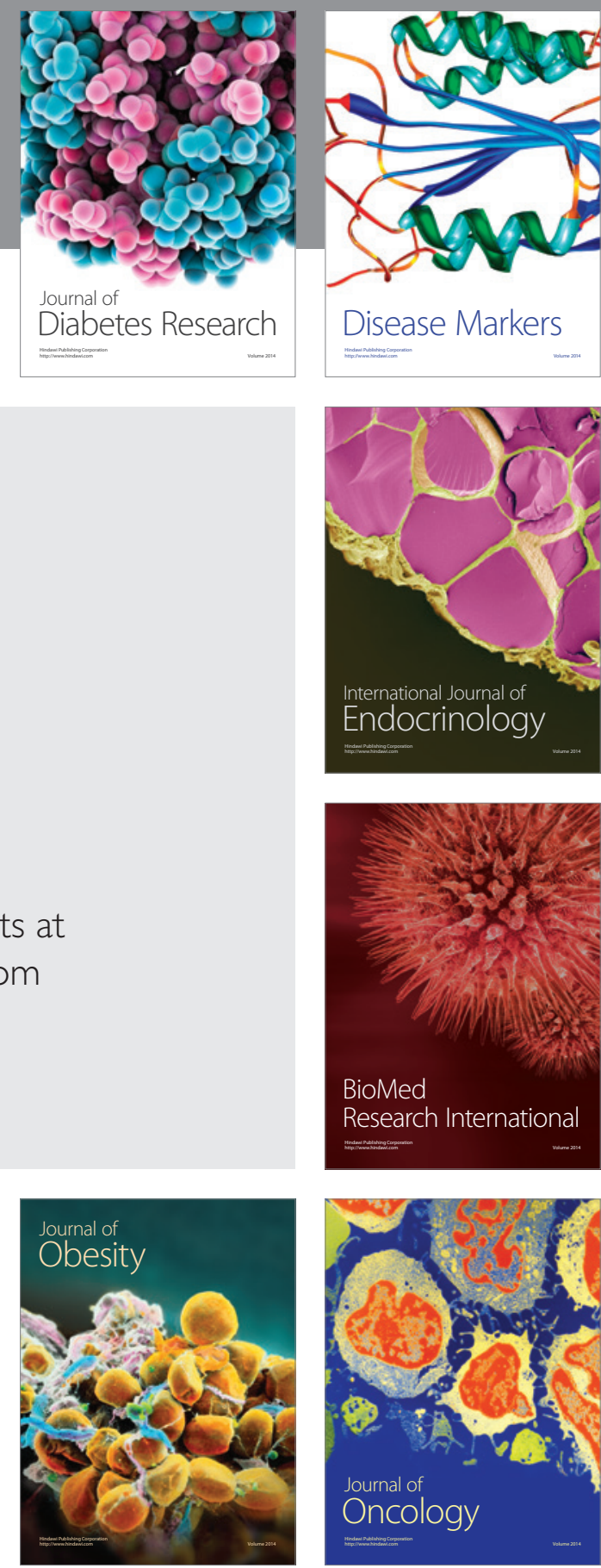

Disease Markers
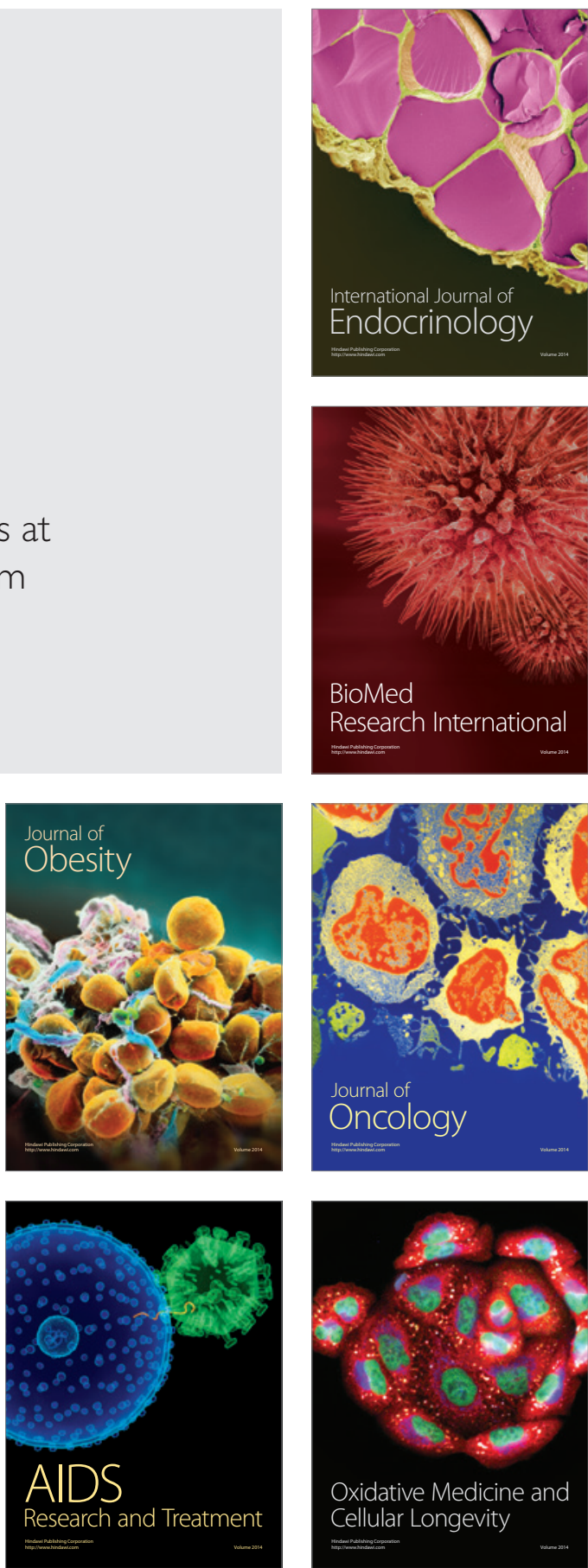